Bull. Korean Math. Soc. 52 (2015), No. 3, pp. 771-788

http://dx.doi.org/10.4134/BKMS.2015.52.3.771

\title{
PARABOLIC MARCINKIEWICZ INTEGRALS ASSOCIATED TO POLYNOMIALS COMPOUND CURVES AND EXTRAPOLATION
}

\author{
Feng Liu And Daiqing Zhang
}

\begin{abstract}
In this note we consider the parametric Marcinkiewicz integrals with mixed homogeneity along polynomials compound curves. Under the rather weakened size conditions on the integral kernels both on the unit sphere and in the radial direction, the $L^{p}$ bounds of such operators are given by an extrapolation argument. Some previous results are greatly extended and improved.
\end{abstract}

\section{Introduction}

Let $\mathbb{R}^{n}(n \geq 2)$ be the $n$-dimensional Euclidean space and $S^{n-1}$ denote the unit sphere in $\mathbb{R}^{n}$ equipped with the induced Lebesgue measure $d \sigma$. Let $\alpha_{j} \geq 1(j=1, \ldots, n)$ be fixed real numbers. Define the function $F: \mathbb{R}^{n} \times$ $(0, \infty) \longrightarrow \mathbb{R}$ by $F(x, \rho)=\sum_{j=1}^{n} x_{j}^{2} \rho^{-2 \alpha_{j}}, x=\left(x_{1}, x_{2}, \ldots, x_{n}\right)$. It is clear that for each fixed $x \in \mathbb{R}^{n}$, the function $F(x, \rho)$ is a decreasing function in $\rho>0$. We let $\rho(x)$ denote the unique solution of the equation $F(x, \rho)=1$. It was showed in [15] that $\left(\mathbb{R}^{n}, \rho\right)$ is a metric space which is often called the mixed homogeneity space related to $\left\{\alpha_{j}\right\}_{j=1}^{n}$. For $\lambda>0$, let $A_{\lambda}$ be the diagonal $n \times n$ matrix $A_{\lambda}=\operatorname{diag}\left\{\lambda^{\alpha_{1}}, \ldots, \lambda^{\alpha_{n}}\right\}$. For a function $\phi: \mathbb{R}^{+} \rightarrow \mathbb{R}^{+}$and $y \in \mathbb{R}^{n}$, we denote $A_{\phi(\rho(y))} y^{\prime}$ by $A_{\phi}(y)$, where $\mathbb{R}^{+}:=(0, \infty)$ and $y^{\prime}=A_{\rho(y)^{-1}} y \in S^{n-1}$.

The change of variables related to the spaces $\left(\mathbb{R}^{n}, \rho\right)$ is given by the transformation

$$
\begin{aligned}
x_{1} & =\rho^{\alpha_{1}} \cos \theta_{1} \cdots \cos \theta_{n-2} \cos \theta_{n-1}, \\
x_{2} & =\rho^{\alpha_{2}} \cos \theta_{1} \cdots \cos \theta_{n-2} \sin \theta_{n-1}, \\
& \vdots \\
x_{n-1} & =\rho^{\alpha_{n-1}} \cos \theta_{1} \sin \theta_{2},
\end{aligned}
$$

Received December 20, 2013; Revised March 27, 2014.

2010 Mathematics Subject Classification. Primary 42B20; Secondary 42B25, 42 B99.

Key words and phrases. Marcinkiewicz integrals, rough kernels, mixed homogeneity, extrapolation.

Supported by the NNSF of China (11101339, 11371295). 


$$
x_{n}=\rho^{\alpha_{n}} \sin \theta_{1} .
$$

Thus $d x=\rho^{\alpha-1} J\left(x^{\prime}\right) d \rho d \sigma\left(x^{\prime}\right)$, where $\rho^{\alpha-1} J\left(x^{\prime}\right)$ is the Jacobian of the above transform and $\alpha=\sum_{j=1}^{n} \alpha_{j}, J\left(x^{\prime}\right)=\sum_{j=1}^{n} \alpha_{j}\left(x_{j}^{\prime}\right)^{2}$. Obviously, $J\left(x^{\prime}\right) \in$ $\mathcal{C}^{\infty}\left(S^{n-1}\right)$ and there exists $M>0$ such that

$$
1 \leq J\left(x^{\prime}\right) \leq M, \quad \forall x^{\prime} \in S^{n-1} .
$$

It is easy to see that

$$
\rho(x)=|x|, \quad \text { if } \alpha_{1}=\alpha_{2}=\cdots=\alpha_{n}=1 .
$$

Let $\Omega$ be integrable on $S^{n-1}$ and satisfy

$$
\begin{gathered}
\int_{S^{n-1}} \Omega(u) J(u) d \sigma(u)=0, \\
\Omega\left(A_{s} x\right)=\Omega(x), \forall s>0 \text { and } x \in \mathbb{R}^{n} .
\end{gathered}
$$

For $d \geq 2$ and a suitable mappings $\Phi: \mathbb{R}^{n} \rightarrow \mathbb{R}^{d}$, we define the parabolic parametric Marcinkiewicz integral operators $\mathscr{M}_{\Omega, h, \Phi}^{\varrho}$ on $\mathbb{R}^{d}$ by

$$
\mathscr{M}_{\Omega, h, \Phi}^{\varrho}(f)(x)=\left(\int_{0}^{\infty}\left|\frac{1}{t^{\varrho}} \int_{\rho(y) \leq t} \frac{\Omega(y) h(\rho(y))}{\rho(y)^{\alpha-\varrho}} f(x-\Phi(y)) d y\right|^{2} \frac{d t}{t}\right)^{1 / 2}
$$

where $f \in \mathscr{S}\left(\mathbb{R}^{d}\right)$ (the Schwartz class), $\varrho=\sigma+i \tau(\sigma, \tau \in \mathbb{R}$ with $\sigma>0)$ and $h \in \Delta_{1}\left(\mathbb{R}^{+}\right)$. Here $\Delta_{\gamma}\left(\mathbb{R}^{+}\right)$for $\gamma \geq 1$ denotes the set of all measurable functions $h$ on $\mathbb{R}^{+}$satisfying the condition

$$
\|h\|_{\Delta_{\gamma}\left(\mathbb{R}^{+}\right)}=\sup _{R>0}\left(R^{-1} \int_{0}^{R}|h(t)|^{\gamma} d t\right)^{1 / \gamma}<\infty .
$$

It is easy to check that $L^{\infty}\left(\mathbb{R}^{+}\right)=\Delta_{\infty}\left(\mathbb{R}^{+}\right) \subsetneq \Delta_{\gamma_{2}}\left(\mathbb{R}^{+}\right) \subsetneq \Delta_{\gamma_{1}}\left(\mathbb{R}^{+}\right)$for $0<\gamma_{1}<\gamma_{2}<\infty$. Also, let $\mathcal{N}_{\alpha}\left(\mathbb{R}^{+}\right), \alpha>0$, be the set of all measurable functions $h$ on $\mathbb{R}^{+}$satisfying $N_{\alpha}(h)=\sum_{m=1} m^{\alpha} 2^{m} d_{m}(h)<\infty$ with $d_{m}(h)=\sup _{k \in \mathbb{Z}} 2^{-k}|E(k, m)|$, where $E(k, 1)=\left\{t \in\left(2^{k}, 2^{k+1}\right]:|h(t)| \leq 2\right\}$, and

$$
E(k, m)=\left\{t \in\left(2^{k}, 2^{k+1}\right]: 2^{m-1}<|h(t)| \leq 2^{m}\right\} \text { for } m \geq 2 .
$$

It follows from $[22]$ that $\Delta_{\gamma}\left(\mathbb{R}^{+}\right) \subsetneq \mathcal{N}_{\alpha}\left(\mathbb{R}^{+}\right)$for any $\alpha>0$ and $1<\gamma<\infty$.

As is well known, the parabolic operators have a long history. It may go back to Fabes and Rivière [15], Madych [20] and Calderón and Torchinsky [6]. If $n=d, h(t)=\varrho=1$ and $\Phi(y)=y$, the operator $\mathscr{M}_{\Omega, h, \Phi}^{\varrho}$ recovers the classical parabolic Marcinkiewicz integral operator denoted by $\mathscr{M}_{\Omega}$, which was discussed extensively by many authors. Xue, Ding and Yabuta [28] first proved that $\mathscr{M}_{\Omega}$ is bounded on $L^{p}\left(\mathbb{R}^{n}\right)$ for $1<p<\infty$, provided that $\Omega \in L^{q}\left(S^{n-1}\right)$ for fixed $q>1$. Afterwards, Chen and Ding [7] (resp., [8]) extended the above result to the case $\Omega \in L\left(\log ^{+} L\right)^{1 / 2}\left(S^{n-1}\right)$ (resp., $\left.\Omega \in H^{1}\left(S^{n-1}\right)\right)$. Moreover, it follows 
from Wang, Chen and Yu's work [25] (also see $[3,19]$ ) that $\mathscr{M}_{\Omega}$ is of type $(p, p)$ for $2 \beta /(2 \beta-1)<p<2 \beta$ if $\Omega \in \mathcal{F}_{\beta}\left(S^{n-1}\right)$ for some $\beta>1$, where

$$
\mathcal{F}_{\beta}\left(S^{n-1}\right):=\left\{\Omega \in L^{1}\left(S^{n-1}\right): \sup _{\xi \in S^{n-1}} \int_{S^{n-1}}\left|\Omega\left(y^{\prime}\right)\right|\left(\log \frac{1}{\left|\xi \cdot y^{\prime}\right|}\right)^{\beta} d \sigma\left(y^{\prime}\right)<\infty\right\}
$$

for all $\beta>0$.

Note that

$$
\begin{aligned}
& \bigcap_{\beta>1} \mathcal{F}_{\beta}\left(S^{n-1}\right) \nsubseteq H^{1}\left(S^{n-1}\right) \nsubseteq \bigcup_{\beta>1} \mathcal{F}_{\beta}\left(S^{n-1}\right) \text { and } \\
& \bigcap_{\beta>1} \mathcal{F}_{\beta}\left(S^{n-1}\right) \nsubseteq L \log ^{+} L\left(S^{n-1}\right) ;
\end{aligned}
$$

$$
\begin{gathered}
L^{q}\left(S^{n-1}\right) \subsetneq L\left(\log ^{+} L\right)\left(S^{n-1}\right) \subsetneq H^{1}\left(S^{n-1}\right) \subsetneq L^{1}\left(S^{n-1}\right) ; \\
L\left(\log ^{+} L\right)^{\beta_{1}}\left(S^{n-1}\right) \subsetneq L\left(\log ^{+} L\right)^{\beta_{2}}\left(S^{n-1}\right), \quad \forall 0<\beta_{2}<\beta_{1} ; \\
L\left(\log ^{+} L\right)^{\beta}\left(S^{n-1}\right) \nsubseteq H^{1}\left(S^{n-1}\right) \nsubseteq L\left(\log ^{+} L\right)^{\beta}\left(S^{n-1}\right), \quad \forall 0<\beta<1 ; \\
L\left(\log ^{+} L\right)^{\beta}\left(S^{n-1}\right) \subsetneq H^{1}\left(S^{n-1}\right), \quad \forall \beta \geq 1 .
\end{gathered}
$$

For the general operator $\mathscr{M}_{\Omega, h, \Phi}^{\varrho}$ in the Euclidean setting, i.e., the case of $\alpha_{1}=\cdots=\alpha_{n}=1$, we denote $\mathscr{M}_{\Omega, h, \Phi}^{\varrho}$ by $\mu_{\Omega, h, \Phi}^{\varrho}$. If $n=d, \Phi(y)=y$ and $h(t)=1$, the operator $\mu_{\Omega, h, \Phi}^{\varrho}$ reduces to the classical parametric Marcinkiewicz integral operator denoted by $\mu_{\Omega}^{\varrho}$. The $L^{p}$ bounds of $\mu_{\Omega}^{\varrho}$ have been discussed extensively by many authors. For example, see $[5,23,24,27]$ for the case $\varrho \equiv 1,[4,17]$ for the case $\varrho>0,[12,21]$ for the case $\varrho \in \mathbb{C}$ with $\operatorname{Re} \varrho>0$. On the other hand, the investigation of the parametric Marcinkiewicz integral operators $\mu_{\Omega, h, \Phi}^{\varrho}$ with rough kernels on the unit sphere as well as in the radial direction have also received a large amount of attention of many authors (see $[2,9,10,11,13]$ et al.). In particular, Al-Qassem and Pan [2] obtained the following result.

Theorem A. Let $\Phi(y)=\mathcal{P}(y)=\left(P_{1}(y), \ldots, P_{d}(y)\right)$ with $P_{j}$ being polynomial on $\mathbb{R}^{n}$. Suppose that $\Omega$ satisfies (1.1)-(1.2) and $\mathcal{P}(y)=-\mathcal{P}(-y)$.

(i) If $\Omega \in L\left(\log ^{+} L\right)^{1 / 2}\left(S^{n-1}\right)$ and $h \in \mathcal{N}_{1 / 2}\left(\mathbb{R}^{+}\right)$, then

$$
\begin{aligned}
& \left\|\mu_{\Omega, h, \Phi}^{\varrho}(f)\right\|_{L^{p}\left(\mathbb{R}^{d}\right)} \\
\leq & C_{p}\left(1+\|\Omega\|_{L\left(\log ^{+} L\right)^{1 / 2}\left(S^{n-1}\right)}\right)\left(1+N_{1 / 2}(h)\right)\|f\|_{L^{p}\left(\mathbb{R}^{d}\right)}, \quad 2 \leq p<\infty ;
\end{aligned}
$$

(ii) If $\Omega \in L\left(\log ^{+} L\right)\left(S^{n-1}\right)$ and $h \in \mathcal{N}_{1}\left(\mathbb{R}^{+}\right)$, then

$\left\|\mu_{\Omega, h, \Phi}^{\varrho}(f)\right\|_{L^{p}\left(\mathbb{R}^{d}\right)} \leq C_{p}\left(1+\|\Omega\|_{L\left(\log ^{+} L\right)\left(S^{n-1}\right)}\right)\left(1+N_{1}(h)\right)\|f\|_{L^{p}\left(\mathbb{R}^{d}\right)}, 1<p<2$.

The constant $C_{p}=C_{\varrho, n, d, p, \max _{1 \leq j \leq d} \operatorname{deg}\left(P_{j}\right)}$ is independent of the coefficients of $P_{j}$ for all $1 \leq j \leq d$. 
In light of the aforementioned facts concerning the above Marcinkiewicz integrals, we find it is natural to ask whether Theorem A holds if replacing $\Phi(y)=\mathcal{P}(y)$ by $\Phi(y)=P(|y|) y^{\prime}$ with $P$ being a polynomial on $\mathbb{R}$. Moreover, another question, which arises from the above result, is the following:

Question 1. For the general case $\alpha_{j} \geq 1(j=1, \ldots, n)$, is $\mathscr{M}_{\Omega, h, \Phi}^{\varrho}$ bounded on $L^{p}\left(\mathbb{R}^{n}\right)$ under the same assumptions on $\Omega$ and $h$ in Theorem $A$, even in the special case $n=d$ and $\Phi(y)=y$ ?

In this paper, we will give an affirmative answer to these questions by the following:

Theorem 1. Let $n=d$ and $\Phi(y)=\left(P_{1}(\varphi(\rho(y))) y_{1}^{\prime}, \ldots, P_{n}(\varphi(\rho(y))) y_{n}^{\prime}\right)$ with $P_{j}$ being real valued polynomials on $\mathbb{R}$ satisfying $P_{j}(0)=0$ and $\varphi \in \mathfrak{F}$. Here, the function class $\mathfrak{F}$ is the set of all function $\varphi$ satisfying the condition (a) or (b).

(a) $\varphi: \mathbb{R}^{+} \rightarrow(0, \infty)$ is a positive increasing $\mathcal{C}^{1}$ function such that $t \varphi^{\prime}(t) \geq$ $C_{\varphi} \varphi(t)$ and $\varphi(2 t) \leq c_{\varphi} \varphi(t)$ for all $t>0$, where $C_{\varphi}$ and $c_{\varphi}$ are independent of $t$.

(b) $\varphi: \mathbb{R}^{+} \rightarrow(0, \infty)$ is a positive decreasing $\mathcal{C}^{1}$ function such that $t \varphi^{\prime}(t) \leq$ $-C_{\varphi} \varphi(t)$ and $\varphi(t) \leq c_{\varphi} \varphi(2 t)$ for all $t>0$, where $C_{\varphi}$ and $c_{\varphi}$ are independent of $t$.

Suppose that $\Omega$ satisfies (1.1)-(1.2).

(i) If $\Omega \in L\left(\log ^{+} L\right)^{1 / 2}\left(S^{n-1}\right)$ and $h \in \mathcal{N}_{1 / 2}\left(\mathbb{R}^{+}\right)$, then

$$
\begin{aligned}
& \left\|\mathscr{M}_{\Omega, h, \Phi}^{\varrho}(f)\right\|_{L^{p}\left(\mathbb{R}^{n}\right)} \\
\leq & C\left(1+\|\Omega\|_{L\left(\log ^{+} L\right)^{1 / 2}\left(S^{n-1}\right)}\right)\left(1+N_{1 / 2}(h)\right)\|f\|_{L^{p}\left(\mathbb{R}^{n}\right)}, \quad 2 \leq p<\infty ;
\end{aligned}
$$

(ii) If $\Omega \in L\left(\log ^{+} L\right)\left(S^{n-1}\right)$ and $h \in \mathcal{N}_{1}\left(\mathbb{R}^{+}\right)$, then

$\left\|\mathscr{M}_{\Omega, h, \Phi}^{\varrho}(f)\right\|_{L^{p}\left(\mathbb{R}^{n}\right)} \leq C\left(1+\|\Omega\|_{L\left(\log ^{+} L\right)\left(S^{n-1}\right)}\right)\left(1+N_{1}(h)\right)\|f\|_{L^{p}\left(\mathbb{R}^{n}\right)}, 1<p<2$.

The constant $C=C_{n, \varrho, p, \max _{1 \leq j \leq n} \operatorname{deg}\left(P_{j}\right), \varphi}$ is independent of the coefficients of $P_{j}$ for all $1 \leq j \leq n$.

Remark 1. There are some model examples in the class $\mathfrak{F}$, such as $t^{\alpha}(\alpha>$ $0), t^{\alpha}(\ln (1+t))^{\beta}(\alpha, \beta>0), t \ln \ln (e+t)$, real-valued polynomials $P$ on $\mathbb{R}$ with positive coefficients and $P(0)=0$ and so on. We note that for any $\varphi \in \mathfrak{F}$, there exists a constant $B_{\varphi}>1$ such that $\varphi(2 t) \geq B_{\varphi} \varphi(t)$ for all $t>0$ if $\varphi$ satisfies the condition (a), and $\varphi(t) \geq B_{\varphi} \varphi(2 t)$ if $\varphi$ satisfies the condition (b) (see $[3,13])$. It should be pointed out that Theorem 1 is also new even for the case $\alpha_{1}=\cdots=\alpha_{n}=1$, in the Euclidean setting.

Remark 2. Theorem 1 essentially improve the result of [1] (see [1, Theorem $1.8]$ ), even in the case $\alpha_{1}=\cdots=\alpha_{n}=1, n=d$ and $P_{1}(t)=\cdots=P_{n}(t)=$ $\varphi(t)=t$. One the other hand, by (1.4) and (1.7), Theorem 1 is distinct from the results of $[13,26]$, even in the case $\alpha_{1}=\cdots=\alpha_{n}=1, n=d$ and $P_{1}(t)=\cdots=P_{n}(t)=\varphi(t)=t$. Moreover, Theorem 1 greatly generalize and 
improve the main result in [5], even in the case $\alpha_{1}=\cdots=\alpha_{n}=1, n=d$ and $P_{1}(t)=\cdots=P_{n}(t)=\varphi(t)=t$.

As several applications of Theorem 1, we have the following corollaries.

Corollary 1. Let $n=d$ and $\Phi(y)=A_{P_{N}(\varphi)}(y)$ with $\varphi \in \mathfrak{F}$, and $P_{N}(t)=$ $\sum_{i=1}^{N} a_{i} t^{i}$ with satisfying $P_{N}(t)>0$ if $t \neq 0$. Suppose that $\Omega$ satisfies (1.1)$(1.2)$.

(i) If $\Omega \in L\left(\log ^{+} L\right)^{1 / 2}\left(S^{n-1}\right)$ and $h \in \mathcal{N}_{1 / 2}\left(\mathbb{R}^{+}\right)$, then

$$
\begin{aligned}
& \left\|\mathscr{M}_{\Omega, h, \Phi}^{\varrho}(f)\right\|_{L^{p}\left(\mathbb{R}^{n}\right)} \\
\leq & C\left(1+\|\Omega\|_{L\left(\log ^{+} L\right)^{1 / 2}\left(S^{n-1}\right)}\right)\left(1+N_{1 / 2}(h)\right)\|f\|_{L^{p}\left(\mathbb{R}^{n}\right)}, 2 \leq p<\infty ;
\end{aligned}
$$

(ii) If $\Omega \in L\left(\log ^{+} L\right)\left(S^{n-1}\right)$ and $h \in \mathcal{N}_{1}\left(\mathbb{R}^{+}\right)$, then

$\left\|\mathscr{M}_{\Omega, h, \Phi}^{\varrho}(f)\right\|_{L^{p}\left(\mathbb{R}^{n}\right)} \leq C\left(1+\|\Omega\|_{L\left(\log ^{+} L\right)\left(S^{n-1}\right)}\right)\left(1+N_{1}(h)\right)\|f\|_{L^{p}\left(\mathbb{R}^{n}\right)}, 1<p<2$.

The constant $C=C_{n, p, \varrho, N, \varphi}$ is independent of the coefficients of $P_{N}$.

Corollary 2. Let $n=d$ and $\alpha_{1}=\cdots=\alpha_{n}=1$. Let $\Phi(y)=P_{N}(\varphi(|y|)) y^{\prime}$ with $\varphi \in \mathfrak{F}$, and $P_{N}(t)=\sum_{i=1}^{N} a_{i} t^{i}$. Suppose that $\Omega$ satisfies (1.1)-(1.2).

(i) If $\Omega \in L\left(\log ^{+} L\right)^{1 / 2}\left(S^{n-1}\right)$ and $h \in \mathcal{N}_{1 / 2}\left(\mathbb{R}^{+}\right)$, then

$$
\begin{aligned}
& \left\|\mu_{\Omega, h, \Phi}^{\varrho}(f)\right\|_{L^{p}\left(\mathbb{R}^{n}\right)} \\
\leq & C\left(1+\|\Omega\|_{L\left(\log ^{+} L\right)^{1 / 2}\left(S^{n-1}\right)}\right)\left(1+N_{1 / 2}(h)\right)\|f\|_{L^{p}\left(\mathbb{R}^{n}\right)}, 2 \leq p<\infty ;
\end{aligned}
$$

(ii) If $\Omega \in L\left(\log ^{+} L\right)\left(S^{n-1}\right)$ and $h \in \mathcal{N}_{1}\left(\mathbb{R}^{+}\right)$, then

$\left\|\mu_{\Omega, h, \Phi}^{\varrho}(f)\right\|_{L^{p}\left(\mathbb{R}^{n}\right)} \leq C\left(1+\|\Omega\|_{L\left(\log ^{+} L\right)\left(S^{n-1}\right)}\right)\left(1+N_{1}(h)\right)\|f\|_{L^{p}\left(\mathbb{R}^{n}\right)}, 1<p<2$.

The constant $C=C_{n, p, \varrho, N, \varphi}$ is independent of the coefficients of $P_{N}$.

The paper is organized as follows. In Section 2 we will present some notations and lemmas. The proof of Theorem 1 will be given in Section 3. We remark that our main methods in the proof of Theorem 1 are taken from [2, 22], but we add some new techniques. Especially, the proofs of (2.6) and (2.29) in this paper are different from [2]. Throughout the paper, we let $p^{\prime}$ denote the conjugate index of $p$ which satisfies $1 / p+1 / p^{\prime}=1$. The letter $C$ will stand for positive constants not necessarily the same one at each occurrence but is independent of the essential variables.

\section{Preliminary lemmas}

Let $N=\max _{1 \leq j \leq n} \operatorname{deg}\left(P_{j}\right)$. For $1 \leq l \leq n$, let $P_{l}(t)=\sum_{i=1}^{N} a_{i, l} t^{i}$. For $1 \leq s \leq N$ and $1 \leq l \leq n$, let $P_{l}^{(s)}(t)=\sum_{i=1}^{s} a_{i, l} t^{i}$ and $P^{(s)}(t)=$ $\left(P_{1}^{(s)}(t), \ldots, P_{n}^{(s)}(t)\right)$. Set $P^{(0)}(t)=0$ and

$$
\Phi_{s}(y)=\left(P_{1}^{(s)}(\varphi(\rho(y))) y_{1}^{\prime}, \ldots, P_{n}^{(s)}(\varphi(\rho(y))) y_{n}^{\prime}\right) .
$$


We can write

$$
\begin{aligned}
\Phi_{s}(y) \cdot \xi & =\sum_{l=1}^{n} \xi_{l} y_{l}^{\prime} P_{l}^{(s)}(\varphi(\rho(y))) \\
& =\sum_{l=1}^{n} \sum_{i=1}^{s} \xi_{l} y_{l}^{\prime} a_{i, l} \varphi(\rho(y))^{i} \\
& =\sum_{i=1}^{s}\left(L_{i}(\xi) \cdot y^{\prime}\right) \varphi(\rho(y))^{i},
\end{aligned}
$$

where $L_{i}: \mathbb{R}^{n} \rightarrow \mathbb{R}^{n}$ is the linear transformation given by

$$
L_{i}(\xi)=\left(a_{i, 1} \xi_{1}, \ldots, a_{i, n} \xi_{n}\right) .
$$

For $1 \leq s \leq N$, let $\lambda_{s}=\operatorname{rank}\left(L_{s}\right)$. By [16, Lemma 6.1], there exist two nonsingular linear transformations $R_{s}: \mathbb{R}^{\lambda_{s}} \rightarrow \mathbb{R}^{\lambda_{s}}$ and $Q_{s}: \mathbb{R}^{n} \rightarrow \mathbb{R}^{n}$ such that

$$
\left|R_{s} \pi_{\lambda_{s}}^{n} Q_{s}(\xi)\right| \leq\left|L_{s}(\xi)\right| \leq C\left|R_{s} \pi_{\lambda_{s}}^{n} Q_{s}(\xi)\right|,
$$

where $C$ depends only on $n$ and $\pi_{\lambda_{s}}^{n}$ is a projection operator from $\mathbb{R}^{n}$ to $\mathbb{R}^{\lambda_{s}}$.

For $1 \leq s \leq N$ and $t>0$, we define the family of measures $\left\{\sigma_{h, t}^{s}\right\}$ and the related maximal operators $\sigma_{h, s}^{*}$ and $M_{h, q, \gamma, s}$ on $\mathbb{R}^{n}$ by

$$
\begin{aligned}
\widehat{\sigma_{h, t}^{s}}(\xi) & =\frac{1}{t^{\varrho}} \int_{t / 2<\rho(y) \leq t} \exp \left(-2 \pi i \xi \cdot \Phi_{s}(y)\right) \frac{h(\rho(y)) \Omega(y)}{\rho(y)^{\alpha-\varrho}} d y ; \\
\sigma_{h, s}^{*}(f)(x) & =\sup _{t \in \mathbb{R}^{+}}|| \sigma_{h, t}^{s}|* f(x)| ; \\
M_{h, q, \gamma, s}(f)(x) & =\sup _{k \in \mathbb{Z}} \int_{2^{q^{\prime} \gamma^{\prime} k}}^{2^{q^{\prime} \gamma^{\prime}(k+1)}}|| \sigma_{h, t}^{s}|* f(x)| \frac{1}{t} d t,
\end{aligned}
$$

where $\left|\sigma_{h, t}^{s}\right|$ is defined in the same way as $\sigma_{h, t}^{s}$, but with $\Omega$ replaced by $|\Omega|$ and $h$ replaced by $|h|$.

The following result follows from Lemmas 2.2 and 2.3 in [18].

Lemma 2.1. Let $\Omega \in L^{q}\left(S^{n-1}\right)$ for some $q>1$ and $P_{\lambda}(t)=\sum_{i=1}^{\lambda} a_{i} t^{i}$ for some $\lambda \in \mathbb{N} \backslash\{0\}$. If $\varphi \in \mathfrak{F}$, then for any $0<\epsilon<\min \left\{1 / q^{\prime}, 1 / \lambda\right\}$ and $\xi \in \mathbb{R}^{n}$, we have

$$
\begin{aligned}
& \int_{r / 2}^{r}\left|\int_{S^{n-1}} \Omega\left(u^{\prime}\right) \exp \left(-i P_{\lambda}(\varphi(t)) \xi \cdot u^{\prime}\right) d \sigma\left(u^{\prime}\right)\right|^{2} \frac{1}{t} d t \\
\leq & C(\varphi)\|\Omega\|_{L^{q}\left(S^{n-1}\right)}^{2}\left|\varphi(r)^{\lambda} a_{\lambda} \xi\right|^{-\epsilon}
\end{aligned}
$$

for $\lambda \in\{1,2, \ldots, N\}$ and any $r>0$. The constant $C(\varphi)$ is independent of $\Omega, q$ and the coefficients of $P_{\lambda}$, but depends on $\varphi$. 
Lemma 2.2. Let $\Omega \in L^{q}\left(S^{n-1}\right)$ satisfying (1.1)-(1.2) and $h \in \Delta_{\gamma}\left(\mathbb{R}^{+}\right)$for some $q, \gamma \in(1,2]$. Suppose that $\varphi \in \mathfrak{F}$. Then for $1 \leq s \leq N$ and $t>0, \xi \in \mathbb{R}^{n}$, there exists a constant $C>0$ such that

$$
\begin{aligned}
\max & \left\{\left\|\sigma_{h, t}^{s}\right\|,\left|\widehat{\sigma_{h, t}^{s}}(\xi)\right|,\left|\widehat{\sigma_{h, t}^{s}}\right|(\xi) \mid\right\} \leq C\|h\|_{\Delta_{\gamma}\left(\mathbb{R}^{+}\right)}\|\Omega\|_{L^{q}\left(S^{n-1}\right)} \\
& \max \left\{\left|\widehat{\sigma_{h, t}^{s}}(\xi)-\widehat{\sigma_{h, t}^{s-1}}(\xi)\right|,\left|\widehat{\sigma_{h, t}^{s} \mid}(\xi)-\widehat{\mid \sigma_{h, t}^{s-1}}\right|(\xi) \mid\right\} \\
\leq & C\|h\|_{\Delta_{\gamma}\left(\mathbb{R}^{+}\right)}\|\Omega\|_{L^{q}\left(S^{n-1}\right)}\left(\varphi(t)^{s}\left|L_{s}(\xi)\right|\right)^{1 /\left(2 s \gamma^{\prime} q^{\prime}\right)} ; \\
& \max \left\{\left|\widehat{\sigma_{h, t}^{s}}(\xi)\right|,\left|\widehat{\sigma_{h, t}^{s} \mid}(\xi)\right|\right\} \\
\leq & C\|h\|_{\Delta_{\gamma}\left(\mathbb{R}^{+}\right)}\|\Omega\|_{L^{q}\left(S^{n-1}\right)}\left(\varphi(t)^{s}\left|L_{s}(\xi)\right|\right)^{-1 /\left(2 s \gamma^{\prime} q^{\prime}\right)} .
\end{aligned}
$$

The constant $C$ is independent of $\Omega, h, q, \gamma$, but depends on $\varphi$.

Proof. Obviously, (2.2) holds. By a change of variable and Hölder's inequality, we have

$$
\begin{aligned}
& \left|\widehat{\sigma_{h, t}^{s}}(\xi)-\widehat{\sigma_{h, t}^{s-1}}(\xi)\right| \\
= & \left|\frac{1}{t^{\varrho}} \int_{t / 2<\rho(y) \leq t}\left(\exp \left(-2 \pi i \xi \cdot \Phi_{s}(y)\right)-\exp \left(-2 \pi i \xi \cdot \Phi_{s-1}(y)\right)\right) \frac{\Omega(y) h(\rho(y))}{\rho(y)^{\alpha-\varrho}} d y\right| \\
\leq & \int_{t / 2<\rho(y) \leq t}\left|L_{s}(\xi) \varphi(\rho(y))^{s}\right| \frac{|\Omega(y) h(\rho(y))|}{\rho(y)^{\alpha}} d y \\
\leq & \int_{t / 2}^{t}|h(r)| \frac{1}{r} d r\|\Omega\|_{L^{1}\left(S^{n-1}\right)} \varphi(t)^{s}\left|L_{s}(\xi)\right| \\
\leq & C\|h\|_{\Delta_{\gamma}\left(\mathbb{R}^{+}\right)}\|\Omega\|_{L^{q}\left(S^{n-1}\right)} \varphi(t)^{s}\left|L_{s}(\xi)\right|
\end{aligned}
$$

which combining with (2.2) implies that

$$
\left|\widehat{\sigma_{h, t}^{s}}(\xi)-\widehat{\sigma_{h, t}^{s-1}}(\xi)\right| \leq C\|h\|_{\Delta_{\gamma}\left(\mathbb{R}^{+}\right)}\|\Omega\|_{L^{q}\left(S^{n-1}\right)}\left(\varphi(t)^{s}\left|L_{s}(\xi)\right|\right)^{1 /\left(2 s \gamma^{\prime} q^{\prime}\right)} .
$$

Similarly,

$$
\left|\widehat{\mid \sigma_{h, t}^{s}}\right|(\xi)-\widehat{\mid \sigma_{h, t}^{s-1}}|(\xi)| \leq C\|h\|_{\Delta_{\gamma}\left(\mathbb{R}^{+}\right)}\|\Omega\|_{L^{q}\left(S^{n-1}\right)}\left(\varphi(t)^{s}\left|L_{s}(\xi)\right|\right)^{1 /\left(2 s \gamma^{\prime} q^{\prime}\right)} .
$$

Thus (2.3) holds. On the other hand, by a change of variable and Hölder's inequality, invoking Lemma 2.1 we have

$$
\begin{aligned}
& \left|\widehat{\sigma_{h, t}^{s}}(\xi)\right| \\
\leq & C \int_{t / 2}^{t}\left|\int_{S^{n-1}} \exp \left(-2 \pi i \xi \cdot \Phi_{s}(y)\right) \Omega\left(y^{\prime}\right) d \sigma\left(y^{\prime}\right)\right||h(r)| \frac{1}{r} d r \\
\leq & C\|h\|_{\Delta_{\gamma}\left(\mathbb{R}^{+}\right)}\|\Omega\|_{L^{1}\left(S^{n-1}\right)}^{1-2 / \gamma^{\prime}}\left(\int_{t / 2}^{t}\left|\int_{S^{n-1}} \Omega\left(y^{\prime}\right) \exp \left(-2 \pi i \xi \cdot \Phi_{s}(y)\right) d \sigma\left(y^{\prime}\right)\right|^{2} \frac{1}{r} d r\right)^{1 / r^{\prime}} \\
\leq & C\|h\|_{\Delta_{\gamma}\left(\mathbb{R}^{+}\right)}\|\Omega\|_{L^{q}\left(S^{n-1}\right)}\left(\varphi(t)^{s}\left|L_{s}(\xi)\right|\right)^{-\epsilon / \gamma^{\prime}}
\end{aligned}
$$


for any $0<\epsilon<\min \left\{1 / q^{\prime}, 1 / s\right\}$. Taking $\epsilon=1 /\left(2 q^{\prime} s\right)$, we have

$$
\left|\widehat{\sigma_{h, t}^{s}}(\xi)\right| \leq C\|\Omega\|_{L^{q}\left(S^{n-1}\right)}\|h\|_{\Delta_{\gamma}\left(\mathbb{R}^{+}\right)}\left(\varphi(t)^{s}\left|L_{s}(\xi)\right|\right)^{-1 /\left(2 s q^{\prime} \gamma^{\prime}\right)} .
$$

Similarly,

$$
\left|\widehat{\sigma_{h, t}^{s}}\right|(\xi) \mid \leq C\|\Omega\|_{L^{q}\left(S^{n-1}\right)}\|h\|_{\Delta_{\gamma}\left(\mathbb{R}^{+}\right)}\left(\varphi(t)^{s}\left|L_{s}(\xi)\right|\right)^{-1 /\left(2 s q^{\prime} \gamma^{\prime}\right)} .
$$

This completes the proof of Lemma 2.2.

Motivated by the idea in [14], we have the following result, which will play a key role in the estimates on some vector-valued norm inequalities.

Lemma 2.3. Let $\Omega, h, \varphi$ be as in Lemma 2.2. Then for $0 \leq s \leq N$ and any $1<p<\infty$, there exists a constant $C>0$ such that

(2.5) $\left\|\sigma_{h, s}^{*}(f)\right\|_{L^{p}\left(\mathbb{R}^{n}\right)} \leq C(q-1)^{-1}(\gamma-1)^{-1}\|h\|_{\Delta_{\gamma}\left(\mathbb{R}^{+}\right)}\|\Omega\|_{L^{q}\left(S^{n-1}\right)}\|f\|_{L^{p}\left(\mathbb{R}^{n}\right)} ;$

$$
\left\|M_{h, q, \gamma, s}(f)\right\|_{L^{p}\left(\mathbb{R}^{n}\right)} \leq C(q-1)^{-1}(\gamma-1)^{-1}\|h\|_{\Delta_{\gamma}\left(\mathbb{R}^{+}\right)}\|\Omega\|_{L^{q}\left(S^{n-1}\right)}\|f\|_{L^{p}\left(\mathbb{R}^{n}\right)} .
$$

The constant $C=C_{n, p, \varphi}$ is independent of $\Omega, h, q, \gamma$ and the coefficients of $P_{j}$ for $1 \leq j \leq n$.

Proof. For convenience, we set $A=(q-1)^{-1}(\gamma-1)^{-1}\|h\|_{\Delta_{\gamma}\left(\mathbb{R}^{+}\right)}\|\Omega\|_{L^{q}\left(S^{n-1}\right)}$. It is easy to verify that

$$
\sigma_{h, s}^{*}(f)(x) \leq \sup _{k \in \mathbb{Z}} \frac{1}{2^{q^{\prime} \gamma^{\prime}(k+1)}} \int_{2^{q^{\prime} \gamma^{\prime} k}<\rho(y) \leq 2^{q^{\prime} \gamma^{\prime}(k+1)}}\left|f\left(x-\Phi_{s}(y)\right)\right| \frac{\mid \Omega(y) h(\rho(y) \mid}{\rho(y)^{\alpha-1}} d y .
$$

For $0 \leq s \leq N$, we define the family of measures $\left\{\mu_{k, s}\right\}$ and maximal operators $\mu_{s}^{*}$ on $\mathbb{R}^{n}$ by

$$
\begin{gathered}
\int_{\mathbb{R}^{n}} f(x) d \mu_{k, s}(x)=\frac{1}{2^{q^{\prime} \gamma^{\prime}(k+1)}} \int_{2^{q^{\prime} \gamma^{\prime} k}<\rho(y) \leq 2^{q^{\prime} \gamma^{\prime}(k+1)}} \frac{\Omega(y) h(\rho(y))}{\rho(y)^{\alpha-1}} f\left(\Phi_{s}(y)\right) d u, \\
\mu_{s}^{*}(f)(x)=\sup _{k \in \mathbb{Z}}|| \mu_{k, s}|* f(x)|,
\end{gathered}
$$

where $\left|\mu_{k, s}\right|$ is defined in the same way as $\mu_{k, s}$, but with $\Omega$ replaced by $|\Omega|$ and $h$ replaced by $|h|$. Thus, we get form (2.7) that

$$
\sigma_{h, s}^{*}(f)(x) \leq \mu_{s}^{*}(|f|)(x) .
$$

Therefore, to prove (2.5), it suffices to prove that

$$
\left\|\mu_{s}^{*}(f)\right\|_{L^{p}\left(\mathbb{R}^{n}\right)} \leq C_{p} A\|f\|_{L^{p}\left(\mathbb{R}^{n}\right)}, 1<p<\infty .
$$

Then by the proof of Lemma 2.2 and a straightforward calculation we get for $1 \leq s \leq N$,

$$
\begin{gathered}
\left|\widehat{\mu_{k, s}}\right|(\xi) \mid \leq C A\left(\min \left\{1,\left(\varphi\left(2^{q^{\prime} \gamma^{\prime} k}\right)^{s}\left|L_{s}(\xi)\right|\right)^{-1}\right\}\right)^{1 /\left(2 s q^{\prime} \gamma^{\prime}\right)} \\
\left|\widehat{\mu_{k, s} \mid}(\xi)-\right| \widehat{\mu_{k, s-1}}|(\xi)| \leq C A\left(\varphi\left(2^{q^{\prime} \gamma^{\prime} k}\right)^{s}\left|L_{s}(\xi)\right|\right)^{1 /\left(2 s q^{\prime} \gamma^{\prime}\right)} .
\end{gathered}
$$

In what follows, we prove $(2.9)$ by induction on $s$. 
Case 1. Without loss of generality, we may assume that $f$ is nonnegative and $\|f\|_{L^{p}\left(\mathbb{R}^{n}\right)}<\infty$, It is easy to check that $\mu_{0}^{*}(f)(x) \leq C A f(x)$, which implies (2.9) for $s=0$.

Case 2. Suppose that (2.9) holds for $s=m-1, m \in\{1, \ldots, N\}$. We shall prove $(2.9)$ for $s=m$. Let $\psi \in \mathcal{C}_{0}^{\infty}(\mathbb{R})$ be supported in $\{|t| \leq 1\}$ and $\psi(t) \equiv 1$ for $|t| \leq 1 / 2$. Define the Borel measures $\left\{\omega_{k, s}\right\}_{k \in \mathbb{Z}}$ on $\mathbb{R}^{n}$ by

$$
\widehat{\omega_{k, m}}(\xi)=\widehat{\mid \mu_{k, m}}\left|(\xi)-\psi\left(\varphi\left(2^{q^{\prime} \gamma^{\prime} k}\right)^{m}\left|R_{m} \pi_{\lambda_{m}}^{n} Q_{m}(\xi)\right|\right)\right| \widehat{\mu_{k, m-1}} \mid(\xi)
$$

for $\xi \in \mathbb{R}^{n}$, where $R_{m}, \pi_{\lambda_{m}}^{n}, Q_{m}$ are as in (2.1). It follows from (2.1) and (2.10)-(2.11) that

$$
\left|\widehat{\omega_{k, m}}(\xi)\right| \leq C A\left(\min \left\{1, \varphi\left(2^{q^{\prime} \gamma^{\prime} k}\right)^{m}\left|L_{m}(\xi)\right|,\left(\varphi\left(2^{q^{\prime} \gamma^{\prime} k}\right)^{m}\left|L_{m}(\xi)\right|\right)^{-1}\right\}\right)^{1 /\left(2 m q^{\prime} \gamma^{\prime}\right)} .
$$

In addition, by (2.13) and a well-known result on maximal functions (see [16]), we have

$$
\begin{aligned}
& \left\|\mu_{m}^{*}(f)\right\|_{L^{p}\left(\mathbb{R}^{n}\right)} \leq\left\|G_{m}(f)\right\|_{L^{p}\left(\mathbb{R}^{n}\right)}+C\left\|\mu_{m-1}^{*}(f)\right\|_{L^{p}\left(\mathbb{R}^{n}\right)}, 1<p<\infty \\
& \left\|\omega_{m}^{*}(f)\right\|_{L^{p}\left(\mathbb{R}^{n}\right)} \leq\left\|G_{m}(f)\right\|_{L^{p}\left(\mathbb{R}^{n}\right)}+C\left\|\mu_{m-1}^{\star}(f)\right\|_{L^{p}\left(\mathbb{R}^{n}\right)}, 1<p<\infty
\end{aligned}
$$

where

$$
\omega_{m}^{*}(f)=\sup _{k \in \mathbb{Z}}|| \omega_{k, m}|* f| \text { and } G_{m}(f)=\left(\sum_{k \in \mathbb{Z}}\left|\omega_{k, m} * f\right|^{2}\right)^{1 / 2} .
$$

By our assumption we have

$$
\left\|\mu_{m-1}^{*}(f)\right\|_{L^{p}\left(\mathbb{R}^{n}\right)} \leq C_{p} A\|f\|_{L^{p}\left(\mathbb{R}^{n}\right)}, 1<p<\infty,
$$

where $C_{p}$ is independent of $q, \gamma$ and the coefficients of $P_{j}$ for all $1 \leq j \leq n$. It remains to prove that

$$
\left\|G_{m}(f)\right\|_{L^{p}\left(\mathbb{R}^{n}\right)} \leq C_{p} A\|f\|_{L^{p}\left(\mathbb{R}^{n}\right)}, 1<p<\infty,
$$

where $C_{p}$ is as above. By a well-known property of Rademacher's functions, (2.17) follows from

$$
\left\|V_{\epsilon}^{m}(f)\right\|_{L^{p}\left(\mathbb{R}^{n}\right)} \leq C_{p, \varphi} A\|f\|_{L^{p}\left(\mathbb{R}^{n}\right)}, 1<p<\infty,
$$

where $V_{\epsilon}^{m}(f)=\sum_{k \in \mathbb{Z}} \epsilon_{k} \omega_{k, m} * f$ with $\epsilon=\left\{\epsilon_{k}\right\}, \epsilon_{k}=1$ or -1 and $C_{p, \varphi}$ is independent of $q, \gamma$ and the coefficients of $\left\{P_{j}\right\}$ for all $1 \leq j \leq n$. Below we prove (2.18). Choose a sequence of nonnegative functions $\left\{\Psi_{k}\right\}_{k \in \mathbb{Z}}$ in $\mathcal{C}_{0}^{\infty}(\mathbb{R})$ such that

$$
\begin{gathered}
\operatorname{supp}\left(\Psi_{k}\right) \subset\left[\varphi\left(2^{q^{\prime} \gamma^{\prime}(k+1)}\right)^{-m}, \varphi\left(2^{q^{\prime} \gamma^{\prime}(k-1)}\right)^{-m}\right], \quad \sum_{k \in \mathbb{Z}} \Psi_{k}^{2}(t)=1, \\
\left|(d / d t)^{j} \Psi_{k}(t)\right| \leq C_{j}|t|^{-j}(j=1,2, \ldots), \quad \forall t>0, j \in \mathbb{N},
\end{gathered}
$$

where $C_{j}$ are independent of $q, \gamma$ and $k$. Define the Fourier multiplier operator $S_{j}$ by

$$
\widehat{S_{j} f}(\xi)=\Psi_{j}\left(\left|R_{m} \pi_{\lambda_{m}}^{n} Q_{m}(\xi)\right|\right) \hat{f}(\xi) \text { for } j \in \mathbb{Z}
$$


Then

$$
\begin{aligned}
V_{\epsilon}^{m}(f) & =\sum_{k \in \mathbb{Z}} \epsilon_{k} \omega_{k, m} * \sum_{j \in \mathbb{Z}} S_{j+k} S_{j+k} f \\
& =\sum_{j \in \mathbb{Z}} \sum_{k \in \mathbb{Z}} \epsilon_{k} S_{j+k}\left(\omega_{k, m} * S_{j+k} f\right):=\sum_{j \in \mathbb{Z}} V_{j}^{m}(f) .
\end{aligned}
$$

By the Littlewood-Paley theory, we have

$$
\left\|V_{j}^{m}(f)\right\|_{L^{p}\left(\mathbb{R}^{n}\right)} \leq C_{p}\left\|\left(\sum_{k \in \mathbb{Z}}\left|\omega_{k, m} * S_{j+k} f\right|^{2}\right)^{1 / 2}\right\|_{L^{p}\left(\mathbb{R}^{n}\right)}, 1<p<\infty .
$$

This combining with Plancherel's theorem yields

$$
\begin{aligned}
\left\|V_{j}^{m}(f)\right\|_{\left(\mathbb{R}^{n}\right)}^{2} & \leq C\left\|\left(\sum_{k \in \mathbb{Z}}\left|\omega_{k, m} * S_{j+k} f\right|^{2}\right)^{1 / 2}\right\|_{L^{2}\left(\mathbb{R}^{n}\right)}^{2} \\
& \leq C \sum_{k \in \mathbb{Z}} \int_{D_{j+k}}\left|\widehat{\omega_{k, m}}(\xi)\right|^{2}|\hat{f}(\xi)|^{2} d \xi
\end{aligned}
$$

where

$$
D_{k}=\left\{\xi \in \mathbb{R}^{n}: \varphi\left(2^{q^{\prime} \gamma^{\prime}(k+1)}\right)^{-m} \leq\left|R_{m} \pi_{\lambda_{m}}^{n} Q_{m}(\xi)\right| \leq \varphi\left(2^{q^{\prime} \gamma^{\prime}(k-1)}\right)^{-m}\right\} .
$$

We get from (2.1), (2.13) and Remark 1 that

$$
\left\|V_{j}^{m}(f)\right\|_{L^{2}\left(\mathbb{R}^{n}\right)} \leq C A\left(B_{\varphi}^{(2-j) / 2} \chi_{\{j \geq 2\}}+B_{\varphi}^{(j+1) / 2} \chi_{\{j<2\}}\right)\|f\|_{L^{2}\left(\mathbb{R}^{n}\right)} .
$$

This together with (2.19) implies

$$
\left\|V_{\epsilon}^{m}(f)\right\|_{L^{2}\left(\mathbb{R}^{n}\right)} \leq C A\|f\|_{L^{2}\left(\mathbb{R}^{n}\right)} .
$$

Thus,

$$
\left\|G_{m}(f)\right\|_{L^{2}\left(\mathbb{R}^{n}\right)} \leq C A\|f\|_{L^{2}\left(\mathbb{R}^{n}\right)},
$$

which combining the Littlewood-Paley theory, (2.13), (2.15)-(2.16) with the proof of Lemma in $[14$, p. 544] implies that

$$
\left\|V_{j}^{m}(f)\right\|_{L^{p}\left(\mathbb{R}^{n}\right)} \leq C_{p} A\|f\|_{L^{p}\left(\mathbb{R}^{n}\right)}, p=4 \text { or } p=4 / 3 .
$$

Interpolating between (2.21) and (2.22) and combining with (2.19), we get

$$
\left\|V_{\epsilon}^{m}(f)\right\|_{L^{p}\left(\mathbb{R}^{n}\right)} \leq C A\|f\|_{L^{p}\left(\mathbb{R}^{n}\right)}, 4 / 3<p<4,
$$

which leads to

$$
\left\|G_{m}(f)\right\|_{L^{p}\left(\mathbb{R}^{n}\right)} \leq C A\|f\|_{L^{p}\left(\mathbb{R}^{n}\right)}, 4 / 3<p<4 .
$$

Reasoning as above, (2.13), (2.15)-(2.16), (2.23), the proof of Lemma in [14, p.544], the Littlewood-Paley theory and interpolation imply

$$
\left\|V_{\epsilon}^{m}(f)\right\|_{L^{p}\left(\mathbb{R}^{n}\right)} \leq C A\|f\|_{L^{p}\left(\mathbb{R}^{n}\right)}, 8 / 7<p<8 .
$$

By using this argument repeatedly, we can obtain ultimately that

$$
\left\|V_{\epsilon}^{m}(f)\right\|_{L^{p}\left(\mathbb{R}^{n}\right)} \leq C A\|f\|_{L^{p}\left(\mathbb{R}^{n}\right)}, 1<p<\infty .
$$

This proves (2.18) and completes the proof of (2.5). 
Below we prove (2.6). It suffices to prove that

$$
\left\|M_{h, q, \gamma, s}(|f|)\right\|_{L^{p}\left(\mathbb{R}^{n}\right)} \leq C_{p} A\|f\|_{L^{p}\left(\mathbb{R}^{n}\right)}
$$

for all $0 \leq s \leq N$ and $1<p<\infty, C_{p}$ is independent of $\Omega, h, q, \gamma$ and the coefficients of $P_{j}$ for all $1 \leq j \leq n$. We shall prove (2.24) by induction on $s$. When $s=0$, it is easy to see that

$$
M_{h, q, \gamma, s}(|f|)(x) \leq C A|f(x)|,
$$

which implies (2.24) for $s=0$. Assume that (2.24) holds for $s=m-1, m \in$ $\{1, \ldots, N\}$, we will prove that $(2.24)$ holds for $s=m$. Let $\psi \in C_{0}^{\infty}(\mathbb{R})$ be as in (2.12). Define the family of measures $\left\{\lambda_{k, m}\right\}_{k \in \mathbb{Z}}$ by

$$
\begin{aligned}
\widehat{\lambda_{k, m}}(\xi)= & \int_{2^{q^{\prime} \gamma^{\prime} k}}^{2^{q^{\prime} \gamma^{\prime}(k+1)}} \widehat{\left|\sigma_{h, t}^{m}\right|}(\xi) \frac{1}{t} d t-\psi\left(\varphi\left(2^{q^{\prime} \gamma^{\prime} k}\right)^{m}\left|R_{m} \pi_{\lambda_{m}}^{n} Q_{m}(\xi)\right|\right) \\
& \times \int_{2^{q^{\prime} \gamma^{\prime} k}}^{2^{q^{\prime} \gamma^{\prime}(k+1)}} \widehat{\mid \sigma_{h, t}^{m-1}} \mid(\xi) \frac{1}{t} d t .
\end{aligned}
$$

By Lemma 2.2 and (2.1), one can easily check that

$$
\left|\widehat{\lambda_{k, m}}(\xi)\right| \leq C A\left(\min \left\{1, \varphi\left(2^{q^{\prime} \gamma^{\prime} k}\right)^{m}\left|L_{m}(\xi)\right|,\left(\varphi\left(2^{q^{\prime} \gamma^{\prime} k}\right)^{m}\left|L_{m}(\xi)\right|\right)^{-1}\right\}\right)^{1 /\left(2 m q^{\prime} \gamma^{\prime}\right)} .
$$

By the definition of $\lambda_{k, m}$ and a well known result on maximal function (see $[16])$, we have

$$
\begin{aligned}
& \left\|M_{h, q, \gamma, m}(|f|)\right\|_{L^{p}\left(\mathbb{R}^{n}\right)} \\
\leq & \left\|g_{m}(|f|)\right\|_{L^{p}\left(\mathbb{R}^{n}\right)}+C\left\|M_{h, q, \gamma, m-1}(|f|)\right\|_{L^{p}\left(\mathbb{R}^{n}\right)}, 1<p<\infty, \\
& \left\|\lambda_{m}^{*}(|f|)\right\|_{L^{p}\left(\mathbb{R}^{n}\right)} \\
\leq & \left\|g_{m}(|f|)\right\|_{L^{p}\left(\mathbb{R}^{n}\right)}+C\left\|M_{h, q, \gamma, m-1}(|f|)\right\|_{L^{p}\left(\mathbb{R}^{n}\right)}, 1<p<\infty,
\end{aligned}
$$

where

$$
g_{m}(f)=\left(\sum_{k \in \mathbb{Z}}\left|\lambda_{k, m} * f\right|^{2}\right)^{1 / 2} \text { and } \lambda_{m}^{*}(f)=\sup _{k \in \mathbb{Z}}|| \lambda_{k, m}|* f| .
$$

From our assumption, (2.26)-(2.28) and the similar arguments as in getting (2.5), we get (2.24) for $s=m$. Thus (2.24) holds. Lemma 2.3 is proved.

Lemma 2.4. Let $h, \Omega, \varphi$ be as in Lemma 2.2. Then for $1 \leq s \leq N$ and any $1<p<\infty$, there exists a constant $C>0$ such that

$$
\begin{gathered}
\quad\left\|\left(\sum_{k \in \mathbb{Z}} \int_{2^{q^{\prime} \gamma^{\prime} k}}^{2^{q^{\prime} \gamma^{\prime}(k+1)}}\left|\sigma_{h, t}^{s} * g_{k}\right|^{2} \frac{1}{t} d t\right)^{1 / 2}\right\|_{L^{p}\left(\mathbb{R}^{n}\right)} \\
\leq C(q-1)^{-1 / 2}(\gamma-1)^{-1 / 2}\|h\|_{\Delta_{\gamma}\left(\mathbb{R}^{+}\right)}\|\Omega\|_{L^{q}\left(S^{n-1}\right)}\left\|\left(\sum_{k \in \mathbb{Z}}\left|g_{k}\right|^{2}\right)^{1 / 2}\right\|_{L^{p}\left(\mathbb{R}^{n}\right)}, 2 \leq p<\infty ;
\end{gathered}
$$




$$
\begin{gathered}
\left\|\left(\sum_{k \in \mathbb{Z}} \int_{2^{q^{\prime} \gamma^{\prime} k}}^{2^{q^{\prime} \gamma^{\prime}(k+1)}}\left|\sigma_{h, t}^{s} * g_{k}\right|^{2} \frac{1}{t} d t\right)^{1 / 2}\right\|_{L^{p}\left(\mathbb{R}^{n}\right)} \\
\leq C(q-1)^{-1}(\gamma-1)^{-1}\|h\|_{\Delta_{\gamma}\left(\mathbb{R}^{+}\right)}\|\Omega\|_{L^{q}\left(S^{n-1}\right)}\left\|\left(\sum_{k \in \mathbb{Z}}\left|g_{k}\right|^{2}\right)^{1 / 2}\right\|_{L^{p}\left(\mathbb{R}^{n}\right)}, 1<p<2 .
\end{gathered}
$$

The constant $C=C_{n, p, \varphi}$ is independent of $\Omega, h, q, \gamma$ and the coefficients of $\left\{P_{j}\right\}$ for all $1 \leq j \leq n$.

Proof. We shall use the method in [2]. First we prove (2.29). For fixed $2 \leq$ $p<\infty$, by duality, there exists a nonnegative function $f$ in $L^{(p / 2)^{\prime}}\left(\mathbb{R}^{n}\right)$ with $\|f\|_{L^{(p / 2)^{\prime}}\left(\mathbb{R}^{n}\right)} \leq 1$ such that

$$
\begin{aligned}
& \left\|\left(\sum_{k \in \mathbb{Z}} \int_{2^{q^{\prime} \gamma^{\prime} k}}^{2^{q^{\prime} \gamma^{\prime}(k+1)}}\left|\sigma_{h, t}^{s} * g_{k}\right|^{2} \frac{1}{t} d t\right)^{1 / 2}\right\|_{L^{p}\left(\mathbb{R}^{n}\right)}^{2} \\
= & \int_{\mathbb{R}^{n}} \sum_{k \in \mathbb{Z}} \int_{2^{q^{\prime} \gamma^{\prime} k}}^{2^{q^{\prime} \gamma^{\prime}(k+1)}}\left|\sigma_{h, t}^{s} * g_{k}\right|^{2} \frac{1}{t} d t f(x) d x .
\end{aligned}
$$

By a change of variable and Hölder's inequality, we obtain

$$
\begin{aligned}
& \left|\sigma_{h, t}^{s} * g_{k}(x)\right|^{2} \\
\leq & \left(\frac{1}{t} \int_{t / 2<\rho(y) \leq t}\left|g_{k}\left(x-\Phi_{s}(y)\right)\right| \frac{|h(\rho(y)) \Omega(y)|}{\rho(y)^{\alpha-1}} d y\right)^{2} \\
\leq & \left(\int_{t / 2}^{t} \int_{S^{n-1}}\left|g_{k}\left(x-\Phi_{s}\left(A_{r} y^{\prime}\right)\right)\right|\left|\Omega\left(y^{\prime}\right)\right| J\left(y^{\prime}\right) d \sigma\left(y^{\prime}\right)|h(r)| \frac{1}{r} d r\right)^{2} \\
\leq & C\|h\|_{\Delta_{\gamma}\left(\mathbb{R}^{+}\right)}^{\gamma}\|\Omega\|_{L^{q}\left(S^{n-1}\right)} \\
& \times\left(\int_{t / 2}^{t} \int_{S^{n-1}}\left|g_{k}\left(x-\Phi_{s}\left(A_{r} y^{\prime}\right)\right)\right|^{2}\left|\Omega\left(y^{\prime}\right)\right| J\left(y^{\prime}\right) d \sigma\left(y^{\prime}\right)|h(r)|^{2-\gamma} \frac{1}{r} d r\right) .
\end{aligned}
$$

Thus by (2.31)-(2.32) and Hölder's inequality, one can check that

$$
\begin{aligned}
& \left\|\left(\sum_{k \in \mathbb{Z}} \int_{2^{q^{\prime} \gamma^{\prime} k}}^{2^{q^{\prime} \gamma^{\prime}(k+1)}}\left|\sigma_{h, t}^{s} * g_{k}\right|^{2} \frac{1}{t} d t\right)^{1 / 2}\right\|_{L^{p}\left(\mathbb{R}^{n}\right)}^{2} \\
\leq & C\|h\|_{\Delta_{\gamma}\left(\mathbb{R}^{+}\right)}^{\gamma}\|\Omega\|_{L^{q}\left(S^{n-1}\right)} \int_{\mathbb{R}^{n}} \sum_{k \in \mathbb{Z}}\left|g_{k}(x)\right|^{2} \tilde{M}_{|h|^{2-\gamma}, q, \gamma, s}(\tilde{f})(-x) d x \\
\leq & C\|h\|_{\Delta_{\gamma}\left(\mathbb{R}^{+}\right)}^{\gamma}\|\Omega\|_{L^{q}\left(S^{n-1}\right)}\left\|\left(\sum_{k \in \mathbb{Z}}\left|g_{k}\right|^{2}\right)^{1 / 2}\right\|_{L^{p}\left(\mathbb{R}^{n}\right)}^{2}\left\|\tilde{M}_{|h|^{2-\gamma}, q, \gamma, s}(\tilde{f})\right\|_{L^{(p / 2)^{\prime}\left(\mathbb{R}^{n}\right)}},
\end{aligned}
$$

where $\tilde{f}(x)=f(-x)$ and $\tilde{M}_{|h|^{2-\gamma}, q, \gamma, s}(f)$ denotes $M_{|h|^{2-\gamma}, q, \gamma, s}$ with $\varrho=1$. Note that $|h(\cdot)|^{2-\gamma} \in \Delta_{\gamma /(2-\gamma)}\left(\mathbb{R}^{+}\right)$, then by (2.6) we have

$$
\left\|\tilde{M}_{|h|^{2-\gamma}, q, \gamma, s}(|\tilde{f}|)\right\|_{L^{(p / 2)^{\prime}\left(\mathbb{R}^{n}\right)}}
$$




$$
\begin{aligned}
& \leq C(q-1)^{-1}(\gamma-1)^{-1}\left\||h|^{2-\gamma}\right\|_{\Delta_{\gamma /(2-\gamma)}\left(\mathbb{R}^{+}\right)}\|\Omega\|_{L^{q}\left(S^{n-1}\right)}\|f\|_{L^{(p / 2)^{\prime}\left(\mathbb{R}^{n}\right)}} \\
& \leq C(q-1)^{-1}(\gamma-1)^{-1}\|h\|_{\Delta_{\gamma}\left(\mathbb{R}^{+}\right)}^{2-\gamma}\|\Omega\|_{L^{q}\left(S^{n-1}\right)},
\end{aligned}
$$

which combining with (2.33) implies (2.29). Next, we prove (2.30). Let $1<$ $p<2$. By duality, there exist functions $\left\{f_{k}(x, t)\right\}$ define on $\mathbb{R}^{n} \times \mathbb{R}^{+}$with

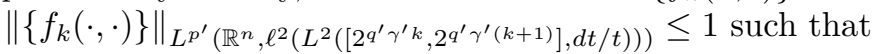

$$
\begin{aligned}
& \left\|\left(\sum_{k \in \mathbb{Z}} \int_{2^{q^{\prime} \gamma^{\prime} k}}^{2^{q^{\gamma^{\prime}}(k+1)}}\left|\sigma_{h, t}^{s} * g_{k}\right|^{2} \frac{1}{t} d t\right)^{1 / 2}\right\|_{L^{p}\left(\mathbb{R}^{n}\right)} \\
\leq & \int_{\mathbb{R}^{n}} \sum_{k \in \mathbb{Z}} \int_{2^{q^{\prime} \gamma^{\prime} k}}^{2^{q^{\prime} \gamma^{\prime}(k+1)}} \sigma_{h, t}^{s} * g_{k}(x) f_{k}(x, t) \frac{1}{t} d t d x \\
\leq & C(q-1)^{-1 / 2}(\gamma-1)^{-1 / 2}\left\|\left(\sum_{k \in \mathbb{Z}}\left|g_{k}\right|^{2}\right)^{1 / 2}\right\|_{L^{p}\left(\mathbb{R}^{n}\right)}\left\|(H(f))^{1 / 2}\right\|_{L^{p^{\prime}}\left(\mathbb{R}^{n}\right)},
\end{aligned}
$$

where

$$
H(f)(x)=\sum_{k \in \mathbb{Z}} \int_{2 q^{\prime} \gamma^{\prime} k}^{2^{q^{\prime} \gamma^{\prime}(k+1)}}\left|\sigma_{h, t}^{s} * \tilde{f}_{k}(x, t)\right|^{2} \frac{1}{t} d t \text { and } \tilde{f}_{k}(x, t)=f(-x, t) .
$$

Since $p^{\prime}>2$, there exists a nonnegative function $u \in L^{\left(p^{\prime} / 2\right)^{\prime}}\left(\mathbb{R}^{n}\right)$ such that

$$
\|H(f)\|_{L^{p^{\prime} / 2\left(\mathbb{R}^{n}\right)}}=\sum_{k \in \mathbb{Z}} \int_{\mathbb{R}^{n}} \int_{2^{q^{\prime} \gamma^{\prime} k}}^{2^{q^{\prime} \gamma^{\prime}(k+1)}}\left|\sigma_{h, t}^{s} * \tilde{f}_{k}(x, t)\right|^{2} \frac{1}{t} d t u(x) d x .
$$

By a similar argument as in (2.32) and (2.5), we have

$$
\begin{aligned}
& \|H(f)\|_{L^{p^{\prime} / 2\left(\mathbb{R}^{n}\right)}} \\
& \leq C\|h\|_{\Delta_{\gamma}\left(\mathbb{R}^{+}\right)}^{\gamma}\|\Omega\|_{L^{q}\left(S^{n-1}\right)} \int_{\mathbb{R}^{n}} \tilde{\sigma}_{|h|^{2-\gamma, s}}^{*}(\tilde{u})(-x)\left(\sum_{k \in \mathbb{Z}} \int_{2^{q^{\prime} \gamma^{\prime} k}}^{2^{q^{\prime} \gamma^{\prime}(k+1)}}\left|\tilde{f}_{k}(x, t)\right|^{2} \frac{1}{t} d t\right) d x \\
& \leq C\|h\|_{\Delta_{\gamma}\left(\mathbb{R}^{+}\right)}^{\gamma}\|S\|_{L^{q}\left(S^{n-1}\right)}\left\|\left(\sum_{k \in \mathbb{Z}} \int_{2^{q^{\prime} \gamma^{\prime} k}}^{2^{q^{\prime} \gamma^{\prime}(k+1)}}\left|f_{k}(x, t)\right|^{2} \frac{1}{t} d t\right)\right\|_{L^{p^{\prime} / 2\left(\mathbb{R}^{n}\right)}}\left\|\tilde{\sigma}_{|h|^{2-\gamma, s}}^{*}(\tilde{u})\right\|_{L^{\left(p^{\prime} / 2\right)^{\prime}\left(\mathbb{R}^{n}\right)}} \\
& \leq C(q-1)^{-1}(\gamma-1)^{-1}\|h\|_{\Delta_{\gamma}\left(\mathbb{R}^{+}\right)}^{2}\|\Omega\|_{L^{q}\left(S^{n-1}\right)}^{2},
\end{aligned}
$$

where $\tilde{u}(x)=u(-x)$ and $\tilde{\sigma}_{|h|^{2-\gamma}, s}^{*}(\tilde{u})$ denotes $\sigma_{|h|^{2-\gamma, s}}^{*}(\tilde{u})$ with $\varrho=1$. (2.30) follows from (2.34) and (2.35). This completes the proof of Lemma 2.4.

\section{Proof of Theorem 1}

This section is devoted to the proof of Theorem 1. We only prove Theorem 1 for the case $\varphi \in \mathfrak{F}$ with satisfying (a), the rest of Theorem 1 can be obtained similarly. Assume that $h \in \Delta_{\gamma}\left(\mathbb{R}^{+}\right)$for some $\gamma \in(1,2]$ and $\Omega \in L^{q}\left(S^{n-1}\right)$ for 
some $q \in(1,2]$ with satisfying (1.1)-(1.2). By Minkowski's inequality, we can write

$$
\begin{aligned}
& \mathscr{M}_{\Omega, h, \Phi}^{\varrho}(f)(x) \\
= & \left(\int_{0}^{\infty}\left|\sum_{k=-\infty}^{0} \frac{1}{t \varrho} \int_{2^{k-1} t<\rho(y) \leq 2^{k} t} f(x-\Phi(y)) \frac{\Omega(y) h(\rho(y))}{\rho(y)^{\alpha-\varrho}} d y\right|^{2} \frac{1}{t} d t\right)^{1 / 2} \\
\leq & \sum_{k=-\infty}^{0}\left(\int_{0}^{\infty}\left|\frac{1}{t^{\varrho}} \int_{2^{k-1} t<\rho(y) \leq 2^{k} t} f(x-\Phi(y)) \frac{\Omega(y) h(\rho(y))}{\rho(y)^{\alpha-\varrho}} d y\right|^{2} \frac{1}{t} d t\right)^{1 / 2} \\
\leq & \left(1-2^{-\sigma}\right)^{-1}\left(\int_{0}^{\infty}\left|\sigma_{h, t}^{N} * f(x)\right|^{2} \frac{1}{t} d t\right)^{1 / 2} .
\end{aligned}
$$

Let $\psi$ be as in (2.12). For $1 \leq s \leq N, t \in \mathbb{R}^{+}$and $\xi \in \mathbb{R}^{n}$, we define the family of measures $\left\{\nu_{t, s}\right\}$ by

$$
\begin{aligned}
\widehat{\nu_{t, s}}(\xi)= & \widehat{\sigma_{h, t}^{s}}(\xi) \prod_{s<j \leq N} \psi\left(\varphi(t)^{j}\left|R_{j} \pi_{\lambda_{j}}^{n} Q_{j}(\xi)\right|\right) \\
& -\widehat{\sigma_{h, t}^{s-1}}(\xi) \prod_{s-1<j \leq N} \psi\left(\varphi(t)^{j}\left|R_{j} \pi_{\lambda_{j}}^{n} Q_{j}(\xi)\right|\right) .
\end{aligned}
$$

It is clear that

$$
\sigma_{h, t}^{N}=\sum_{s=1}^{N} \nu_{t, s}
$$

Here we use the convention $\Pi_{j \in \emptyset} a_{j}=1$. By Lemma 2.1 and (2.1), we get (3.4)

$$
\begin{aligned}
& \left|\widehat{\nu_{t, s}}(\xi)\right| \\
\leq & C\|h\|_{\Delta_{\gamma}\left(\mathbb{R}^{+}\right)}\|\Omega\|_{L^{q}\left(S^{n-1}\right)} \min \left\{1, \varphi(t)^{s}\left|L_{s}(\xi)\right|,\left(\varphi(t)^{s}\left|L_{s}(\xi)\right|\right)^{-1}\right\}^{1 /\left(2 s \gamma^{\prime} q^{\prime}\right)}
\end{aligned}
$$

for $1 \leq s \leq N$. The constant $C$ is independent of $\Omega, h, q, \gamma$ and the coefficients of $P_{j}$ for all $1 \leq j \leq n$. This together with a straightforward calculation yields that

$$
\begin{aligned}
& \left(\int_{2^{q^{\prime} \gamma^{\prime} k}}^{2^{q^{\prime} \gamma^{\prime}(k+1)}}\left|\widehat{\nu_{t, s}}(\xi)\right|^{2} \frac{1}{t} d t\right)^{1 / 2} \\
& \leq C(q-1)^{-1 / 2}(\gamma-1)^{-1 / 2}\|h\|_{\Delta_{\gamma}\left(\mathbb{R}^{+}\right)}\|\Omega\|_{L^{q}\left(S^{n-1}\right)} \\
& \times \min \left\{1, \varphi\left(2^{\gamma^{\prime} q^{\prime} k}\right)^{s}\left|L_{s}(\xi)\right|,\left(\varphi\left(2^{\gamma^{\prime} q^{\prime} k}\right)^{s}\left|L_{s}(\xi)\right|\right)^{-1}\right\}^{1 /\left(2 s \gamma^{\prime} q^{\prime}\right)},
\end{aligned}
$$

where $C$ is independent of $\Omega, h, q, \gamma$ and the coefficients of $P_{j}$ for all $1 \leq j \leq n$. It follows from (3.1), (3.3) and Minkowski's inequality that

$$
\mathscr{M}_{\Omega, h, \Phi}^{\varrho}(f)(x) \leq C \sum_{s=1}^{N}\left(\int_{0}^{\infty}\left|\nu_{t, s} * f(x)\right|^{2} \frac{1}{t} d t\right)^{1 / 2}:=C \sum_{s=1}^{N} \mathscr{M}_{s}(f)(x) .
$$


Choose a sequence of nonnegative functions $\left\{\Psi_{k}\right\}_{k \in \mathbb{Z}}$ in $\mathcal{C}_{0}^{\infty}(\mathbb{R})$ such that

$$
\begin{aligned}
& \operatorname{supp}\left(\Psi_{k}\right) \subset\left[\varphi\left(2^{q^{\prime} \gamma^{\prime}(k+1)}\right)^{-s}, \varphi\left(2^{q^{\prime} \gamma^{\prime}(k-1)}\right)^{-s}\right], \quad \sum_{k \in \mathbb{Z}} \Psi_{k}(t)=1, \\
& \left|(d / d t)^{j} \Psi_{k}(t)\right| \leq C_{j}|t|^{-j}(j=1,2, \ldots) \text { for all } t>0 \text { and } j \in \mathbb{N},
\end{aligned}
$$

where $C_{j}$ are independent of $q, \gamma, k$. Define the Fourier multiplier operator $\Gamma_{j}$ by

$$
\widehat{\Gamma_{j}(f)}(\xi)=\Psi_{j}\left(\left|R_{s} \pi_{\lambda_{s}}^{n} Q_{s}(\xi)\right|\right) \hat{f}(\xi) \text { for } j \in \mathbb{Z}
$$

Then we have

$$
\mathscr{M}_{s}(f)(x) \leq \sum_{i \in \mathbb{Z}} \mathscr{M}_{s, i}(f)(x)
$$

where

$$
\mathscr{M}_{s, i}(f)(x)=\left(\sum_{k \in \mathbb{Z}} \int_{2^{q^{\prime} \gamma^{\prime} k}}^{2^{q^{\prime} \gamma^{\prime}(k+1)}}\left|\nu_{t, s} * \Gamma_{i+k} f(x)\right|^{2} \frac{1}{t} d t\right)^{1 / 2} .
$$

Below we estimate the $L^{p}$ estimates for $\mathscr{M}_{s, i}$. By Lemma 2.4 and the definition of $\nu_{t, s}$, we have for $1 \leq s \leq N$, there exists a positive constant $C=C_{n, p, \varphi}$, which is independent of $\Omega, h, q, \gamma$ such that

$$
\begin{aligned}
& \left\|\left(\sum_{k \in \mathbb{Z}} \int_{2^{q^{\prime} \gamma^{\prime} k}}^{2^{q^{\prime} \gamma^{\prime}(k+1)}}\left|\nu_{t, s} * g_{k}\right|^{2} \frac{1}{t} d t\right)^{1 / 2}\right\| \|_{L^{p}\left(\mathbb{R}^{n}\right)} \\
\leq & C(q-1)^{-1 / 2}(\gamma-1)^{-1 / 2}\|h\|_{\Delta_{\gamma}\left(\mathbb{R}^{+}\right)}\|\Omega\|_{L^{q}\left(S^{n-1}\right)}\left\|\left(\sum_{k \in \mathbb{Z}}\left|g_{k}\right|^{2}\right)^{1 / 2}\right\|_{L^{p}\left(\mathbb{R}^{n}\right)}, 2 \leq p<\infty ;
\end{aligned}
$$

$$
\begin{gathered}
\left\|\left(\sum_{k \in \mathbb{Z}} \int_{2^{q^{\prime} \gamma^{\prime} k}}^{2^{q^{\prime} \gamma^{\prime}(k+1)}}\left|\nu_{t, s} * g_{k}\right|^{2} \frac{1}{t} d t\right)^{1 / 2}\right\|_{L^{p}\left(\mathbb{R}^{n}\right)} \\
\leq C(q-1)^{-1}(\gamma-1)^{-1}\|h\|_{\Delta_{\gamma}\left(\mathbb{R}^{+}\right)}\|\Omega\|_{L^{q}\left(S^{n-1}\right)}\left\|\left(\sum_{k \in \mathbb{Z}}\left|g_{k}\right|^{2}\right)^{1 / 2}\right\|_{L^{p}\left(\mathbb{R}^{n}\right)}, 1<p<2 .
\end{gathered}
$$

By (3.9)-(3.10) and the Littlewood-Paley theory, we have

$$
\begin{aligned}
& \left\|\mathscr{M}_{s, i}(f)\right\|_{L^{p}\left(\mathbb{R}^{n}\right)} \\
= & \left\|\left(\sum_{k \in \mathbb{Z}} \int_{2^{q^{\prime} \gamma^{\prime} k}}^{2^{q^{\prime} \gamma^{\prime}(k+1)}}\left|\nu_{t, s} * \Gamma_{i+k} f(x)\right|^{2} \frac{1}{t} d t\right)^{1 / 2}\right\|_{L^{p}\left(\mathbb{R}^{n}\right)} \\
\leq & C(q-1)^{-1 / 2}(\gamma-1)^{-1 / 2}\|h\|_{\Delta_{\gamma}\left(\mathbb{R}^{+}\right)}\|\Omega\|_{L^{q}\left(S^{n-1}\right)}\left\|\left(\sum_{k \in \mathbb{Z}}\left|\Gamma_{i+k} f\right|^{2}\right)^{1 / 2}\right\| L_{L^{p}\left(\mathbb{R}^{n}\right)} \\
\leq & C(q-1)^{-1 / 2}(\gamma-1)^{-1 / 2}\|h\|_{\Delta_{\gamma}\left(\mathbb{R}^{+}\right)}\|\Omega\|_{L^{q}\left(S^{n-1}\right)}\|f\|_{L^{p}\left(\mathbb{R}^{n}\right)}, \quad 2 \leq p<\infty .
\end{aligned}
$$


Similarly,

$$
\begin{aligned}
& \left\|\mathscr{M}_{s, i}(f)\right\|_{L^{p}\left(\mathbb{R}^{n}\right)} \\
\leq & C(q-1)^{-1}(\gamma-1)^{-1}\|h\|_{\Delta_{\gamma}\left(\mathbb{R}^{+}\right)}\|\Omega\|_{L^{q}\left(S^{n-1}\right)}\|f\|_{L^{p}\left(\mathbb{R}^{n}\right)}, \quad 1<p<2 .
\end{aligned}
$$

On the other hand, by (2.1), (3.5), Remark 1 and Plancherel's theorem, we have

$$
\begin{aligned}
& \left\|\mathscr{M}_{s, i}(f)\right\|_{L^{2}\left(\mathbb{R}^{n}\right)}^{2} \\
= & \sum_{k \in \mathbb{Z}} \int_{\mathbb{R}^{n}}\left|\Psi_{i+k}\left(\left|R_{s} \pi_{\lambda_{s}}^{n} Q_{s}(\xi)\right|\right)\right|^{2}|\hat{f}(\xi)|^{2}\left(\int_{2^{q^{\prime} \gamma^{\prime} k}}^{2^{q^{\prime} \gamma^{\prime}(k+1)}}\left|\widehat{\nu_{t, s}}(\xi)\right|^{2} \frac{1}{t} d t\right) d \xi \\
\leq & \sum_{k \in \mathbb{Z}} \int_{E_{i+k}}\left(\int_{2^{q^{\prime} \gamma^{\prime} k}}^{2^{q^{\prime} \gamma^{\prime}(k+1)}}\left|\widehat{\nu_{t, s}}(\xi)\right|^{2} \frac{1}{t} d t\right)|\hat{f}(\xi)|^{2} d \xi \\
\leq & C(q-1)^{-1}(\gamma-1)^{-1}\|h\|_{\Delta_{\gamma}\left(\mathbb{R}^{+}\right)}^{2}\|\Omega\|_{L^{q}\left(S^{n-1}\right)}^{2} D_{i}^{2}\|f\|_{L^{2}\left(\mathbb{R}^{n}\right)}^{2},
\end{aligned}
$$

where $D_{i}=B_{\varphi}^{(2-i) / 2} \chi_{\{i \geq 2\}}+B_{\varphi}^{i / 2} \chi_{\{i<2\}}$ and

$$
E_{i+k}=\left\{\xi \in \mathbb{R}^{n}: \varphi\left(2^{\gamma^{\prime} q^{\prime}(i+k+1)}\right)^{-s} \leq\left|R_{s} \pi_{\lambda_{s}}^{n} Q_{s}(\xi)\right| \leq \varphi\left(2^{\gamma^{\prime} q^{\prime}(i+k-1)}\right)^{-s}\right\} .
$$

Thus

$$
\begin{aligned}
& \left\|\mathscr{M}_{s, i}(f)\right\|_{L^{2}\left(\mathbb{R}^{n}\right)} \\
\leq & C(q-1)^{-1 / 2}(\gamma-1)^{-1 / 2}\|h\|_{\Delta_{\gamma}\left(\mathbb{R}^{+}\right)}\|\Omega\|_{L^{q}\left(S^{n-1}\right)} D_{i}\|f\|_{L^{2}\left(\mathbb{R}^{n}\right)} .
\end{aligned}
$$

By interpolation between (3.11) and (3.14) leads to (3.15)

$$
\left\|\mathscr{M}_{s, i}(f)\right\|_{L^{p}\left(\mathbb{R}^{n}\right)}
$$

$$
\leq C(q-1)^{-1 / 2}(\gamma-1)^{-1 / 2}\|h\|_{\Delta_{\gamma}\left(\mathbb{R}^{+}\right)}\|\Omega\|_{L^{q}\left(S^{n-1}\right)} D_{i}^{\alpha_{p}}\|f\|_{L^{p}\left(\mathbb{R}^{n}\right)}, \quad 2 \leq p<\infty .
$$

The constant $\alpha_{p}$ depends only on $p$. It follows from (3.8) and (3.15) that (3.16)

$$
\left\|\mathscr{M}_{s}(f)\right\|_{L^{p}\left(\mathbb{R}^{n}\right)}
$$

$$
\leq C(q-1)^{-1 / 2}(\gamma-1)^{-1 / 2}\|h\|_{\Delta_{\gamma}\left(\mathbb{R}^{+}\right)}\|\Omega\|_{L^{q}\left(S^{n-1}\right)}\|f\|_{L^{p}\left(\mathbb{R}^{n}\right)}, 2 \leq p<\infty .
$$

Similarly, by (3.8) and interpolation between (3.12) and (3.14), we have

$$
\begin{aligned}
& \left\|\mathscr{M}_{s}(f)\right\|_{L^{p}\left(\mathbb{R}^{n}\right)} \\
\leq & C(q-1)^{-1}(\gamma-1)^{-1}\|h\|_{\Delta_{\gamma}\left(\mathbb{R}^{+}\right)}\|\Omega\|_{L^{q}\left(S^{n-1}\right)}\|f\|_{L^{p}\left(\mathbb{R}^{n}\right)}, \quad 1<p<2 .
\end{aligned}
$$

Using (3.6), (3.16)-(3.17), we get

$$
\begin{aligned}
& \left\|\mathscr{M}_{\Omega, h, \Phi}^{\varrho}(f)\right\|_{L^{p}\left(\mathbb{R}^{n}\right)} \\
& \leq C(q-1)^{-1 / 2}(\gamma-1)^{-1 / 2}\|h\|_{\Delta_{\gamma}\left(\mathbb{R}^{+}\right)}\|\Omega\|_{L^{q}\left(S^{n-1}\right)}\|f\|_{L^{p}\left(\mathbb{R}^{n}\right)}, 2 \leq p<\infty, \\
& \quad\left\|\mathscr{M}_{\Omega, h, \Phi}^{\varrho}(f)\right\|_{L^{p}\left(\mathbb{R}^{n}\right)} \\
& \quad \leq C(q-1)^{-1}(\gamma-1)^{-1}\|h\|_{\Delta_{\gamma}\left(\mathbb{R}^{+}\right)}\|\Omega\|_{L^{q}\left(S^{n-1}\right)}\|f\|_{L^{p}\left(\mathbb{R}^{n}\right)}, 1<p<2 .
\end{aligned}
$$


The constant $C=C_{\varrho, n, p, N, \varphi}$ is independent of $\Omega, h, q, \gamma$. Finally, Theorem 1 follows directly from (3.18)-(3.19) and an extrapolation argument as in the proof of [22, Theorem 1.2].

Acknowledgement. The authors would like to express their deep gratitude to the referee for his/her careful reading, valuable comments and suggestions.

\section{References}

[1] H. Al-Qassem, L. C. Cheng, and Y. Pan, On certain rough integral operators and extrapolation, J. Inequal. Pure Appl. Math. 10 (2009), no. 3, Article 78, 15 pp.

[2] H. Al-Qassem and Y. Pan, On certain estimates for Marcinkiewicz integrals and extrapolation, Collect. Math. 60 (2009), no. 2, 123-145.

[3] A. Al-Salman, A note on parabolic Marcinkiewicz integrals along surfaces, Proc. A. Razmadze Math. Inst. 154 (2010), 21-36.

[4] , On the $L^{2}$ boundedness of parametric Marcinkiewicz integral operator, J. Math. Anal. Appl. 375 (2011), no. 2, 745-752.

[5] A. Al-Salman, H. Al-Qassem, L. C. Cheng, and Y. Pan, L ${ }^{p}$ bounds for the function of Marcinkiewicz, Math. Res. Lett. 9 (2002), no. 5-6, 697-700.

[6] A. P. Calderón and A. Torchinsky, Parabolic maximal functions associated with a distribution, Adv. in Math. 16 (1975), 1-64.

[7] Y. Chen and Y. Ding, $L^{p}$ bounds for the parabolic Marcinkiewicz integral with rough kernels, J. Korean Math. Soc. 44 (2007), no. 3, 733-745.

[8] — The parabolic Littlewood-Paley operator with Hardy space kernels, Canad. Math. Bull. 52 (2009), no. 4, 521-534.

[9] Y. Ding, D. Fan, and Y. Pan, $L^{p}$-boundedness of Marcinkiewicz integrals with Hardy space function kernel, Acta Math. Sin. (Engl. Ser.) 16 (2000), no. 4, 593-600.

[10] (2002), no. 1, 17-26.

[11] Y. Ding, S. Lu, and K. Yabuta, A problem on rough parametric Marcinkiewicz functions, J. Aust. Math. Soc. 72 (2002), no. 1, 13-21.

[12] Y. Ding, Q. Xue, and K. Yabuta, A remark to the $L^{2}$ boundedness of parametric Marcinkiewicz integral, J. Math. Anal. Appl. 387 (2012), no. 2, 691-697.

[13] _ Boundedness of the Marcinkiewicz integrals with rough kernel associated to surfaces, Tohoku Math. J. 62 (2010), no. 2, 233-262.

[14] J. Duoandikoetxea and J. L. Rubio de Francia, Maximal and singular integral operators via Fourier transform estimates, Invent. Math. 84 (1986), 541-561.

[15] E. Fabes and N. Rivière, Singular integrals with mixed homogeneity, Studia Math. 27 (1966), 19-38.

[16] D. Fan and Y. Pan, Singular integral operators with rough kernels supported by subvarieties, Amer. J. Math. 119 (1997), no. 4, 799-839.

[17] L. Hörmander, Estimates for translation invariant operators in $L^{p}$ spaces, Acta Math. 104 (1960), 93-104

[18] F. Liu, On singular integrals associated to surfaces, Tohoku Math. J. 66 (2014), no. 1, $1-14$.

[19] F. Liu and H. Wu, Rough Marcinkiewicz integrals with mixed homogeneity on product spaces, Acta Math. Sin. (Engl. Ser.) 29 (2013), no. 7, 1231-1244.

[20] W. R. Madych, On Littlewood-Paley functions, Studia Math. 50 (1974), 43-63.

[21] M. Sakamoto and K. Yabuta, Boundedness of Marcinkiewicz functions, Studia Math. 135 (1999), no. 2, 103-142.

[22] S. Sato, Estimates for singular integrals and extrapolation, Studia Math. 192 (2009), no. 3, 219-233. 
[23] E. M. Stein, On the function of Littlewood-Paley, Lusin and Marcinkiewicz, Trans. Amer. Math. Soc. 88 (1958), 430-466.

[24] T. Walsh, On the function of Marcinkiewicz, Studia Math. 44 (1972), 203-217.

[25] F. Wang, Y. Chen, and W. Yu, $L^{p}$ bounds for the parabolic Littlewood-Paley operator associated to surfaces of revolution, Bull. Korean Math. Soc. 49 (2012), no. 4, 787-797.

[26] H. Wu, On Marcinkiewicz integral operator with rough kernels, Integral Equations Operator Theory 52 (2005), no. 2, 285-298.

[27] _ $L^{p}$ bounds for Marcinkiewicz integrals associates to surfaces of revolution, J. Math. Anal. Appl. 321 (2006), no. 2, 811-827.

[28] Q. Xue, Y. Ding, and K. Yabuta, Parabolic Littlewood-Paley g-function with rough kernel, Acta Math. Sin. (Engl. Ser.) 24 (2008), no. 12, 2049-2060.

Feng LiU

College of Mathematics and Systems Science

Shandong University of Science and Technology

Qingdao, Shandong 266590, P. R. China

E-mail address: liufeng860314@163.com

DAIQING ZHANG

School of Mathematical Sciences

Xiamen University

Xiamen, Fujian 361005, P. R. China

E-mail address: zhangdaiqing2011@163.com 